\title{
BMJ Open Quality Delivery of remote otology care: a UK pilot feasibility study
}

\author{
Cillian T Forde (D) , ${ }^{1}$ Lilia Dimitrov (1),${ }^{1,2,3}$ Suneal Doal, ${ }^{4}$ Jay Patel, ${ }^{4}$ Dawn Clare, ${ }^{4}$ \\ Michael Burslem, ${ }^{1}$ Nishchay Mehta, ${ }^{1,2,3}$ Joseph G Manjaly ${ }^{1,2,3}$
}

To cite: Forde CT,

Dimitrov L, Doal S, et al. Delivery of remote otology care: a UK pilot feasibility study. BMJ Open Quality 2022;11:e001444. doi:10.1136/ bmjoq-2021-001444

- Additional supplemental material is published online only. To view, please visit the journal online (http://dx.doi.org/10. 1136/bmjoq-2021-001444).

CTF and LD contributed equally.

CTF and LD are joint first authors.

Received 27 February 2021 Accepted 14 September 2021

Check for updates

(C) Author(s) (or their employer(s)) 2022. Re-use permitted under CC BY-NC. No commercial re-use. See rights and permissions. Published by BMJ.

${ }^{1}$ ENT Department, Royal National Throat Nose and Ear Hospital, London, UK ${ }^{2}$ evidENT Ucl Ear Institute, University College London, London, UK

${ }^{3}$ National Institute of Health Research University College London Hospitals Biomedical Research Centre, London, UK ${ }^{4}$ Department of Audiology, Royal National ENT \& Eastman Dental Hospitals, London, UK

Correspondence to

Dr Joseph G Manjaly;

joseph.manjaly@nhs.net

\section{ABSTRACT}

Introduction The COVID-19 pandemic has catalysed the need to implement the National Health Service Long-Term Plan to deliver more care in the community and to reduce face-to-face hospital appointments by up to $33 \%$. This study aimed to assess the feasibility of a remote otology service from triage through to delivery.

Methods New adult otology referrals at a tertiary ear, nose and throat (ENT) hospital aged between 18 and 70 with hearing loss or tinnitus were included. Patients attended an audiology-led community clinic where they underwent a focused history, audiometric testing, and a smartphone-based application and otoscope (Tympa System) was used to capture still and video images of their eardrums. The information was reviewed by ENT clinicians using a remote review platform with a subset of patients subsequently undergoing an in-person review to measure concordance between the two assessments.

Results 58 patients participated. $75 \%$ of patients had their pathways shortened by one hospital visit with $65 \%$ avoiding any hospital attendances. $24 \%$ required an additional face-to-face appointment due to incomplete views of the tympanic membrane or need for additional examinations. Electronic validation by a blinded consultant otologist demonstrated a diagnosis concordance of $95 \%$, and concordance between remote-review and in-person consultations in the 12 patients who agreed to attend for an in-person review was $83.3 \%$. $98 \%$ of patients were satisfied with the pathway.

Conclusion This pilot service is feasible, safe and noninferior to the traditional outpatient model in the included patient group. There is potential for the development of a community audiology-led service or use for general practioner advice and guidance.

\section{INTRODUCTION}

In the UK in 2018-2019, there were over 3 million ear, nose and throat (ENT) outpatient appointments and 1.4 million audiology appointments. ${ }^{12}$ Only $0.13 \%$ of initial appointments and $1.06 \%$ of follow-ups were conducted as teleconsultations. ${ }^{1}{ }^{2}$ Otological and hearing reports form a significant proportion of those referred to ENT from primary care.

The National Health Service (NHS) LongTerm Plan aims to redirect care to the community and reduce face-to-face appointments by up to $33 \%$ over 5 years. ${ }^{1}$ It is anticipated that a shift towards community-delivered healthcare
What are the new findings

- We describe an innovative pilot service model that can be used across the whole patient pathway to deliver remote otological care.

- Our pilot has demonstrated safety and non-inferiority to a traditional hospital-based otology service for the selected group of patients.

- Shortened hospital pathway and reduced footfall.

- Good patient satisfaction with the remote review pathway.

How might it impact on healthcare in the future

Potential for the development of a community audiology-led service.

- Potential for use as general practioner advice and guidance.

- Potential for use as part of an audiology-led cholesteatoma surveillance clinic.

- Allowing for the use of microsuction in future uses of this pathway, more patients could be treated in the community than we demonstrated.

will optimise the patient experience as well as reducing travel-related carbon emissions and congestion on public transport. Digital transformation is one of the major strategies in this process.

Advances in the production of portable medical devices that can capture endoscopicgrade images of the tympanic membrane offer opportunities for the delivery of specialist care outside of the traditional hospital-based setting. ${ }^{1}$ The term teleotology has been coined to describe this model. In the literature, it has been used as an aid for general practioners (GPs) ${ }^{34}$ as well as a means for care delivery to patients in rural locations with limited access to ENT services. ${ }^{4-7}$

The COVID-19 pandemic has accelerated the need for alternative otology care pathways away from the traditional model of an audiology hearing assessment followed by ENT review for examination with otoscopy and treatment. Among the significant upheaval, it 
has also been a time of great innovation, and opportunities have emerged to address long-standing issues in both the delivery of care and organisation of services.

In March 2020, the Health Protection (Coronavirus, Restrictions) (England) Regulations 2020 came into force legally mandating the population in England to stay at home unless necessary. This has come to be known as the first National Lockdown. In response to this, ENT UK published guidance to keep elective outpatient attendances 'to the safe minimum and increasing the use of telephone clinics where possible'. ${ }^{8}$ A consequence of this response was that appointments requiring a physical consultation were postponed, leading to a large backlog of otology patients awaiting review. Furthermore, with resumption of services, new COVID-19-related restraints, including reduced clinic numbers, restrictions on patient flow and patient numbers in waiting area spaces, further lengthened waiting lists.

We explored an alternative option for specialist treatment that would deliver the key elements of an otological consultation (history, examination and hearing test) while reducing footfall in the hospital. The use of technology to provide an image-based diagnostic service has been reported during COVID-19; however, to the best of our knowledge, this is the first description of an otology service encompassing the full pathway from triage in the $\mathrm{UK}^{9}$

The aim of this study was to assess the feasibility of a teleotology or remote otology consultation service from triage through to service delivery.

\section{MATERIALS AND METHODS \\ Context}

Patients referred for otology services in North Central London attend specialist clinics at the Royal National ENT Hospital in central London. Most patients are seen by an audiologist for hearing assessment and then an ENT surgeon for a history, otoscopy and management plan. The teleotology pathway was designed to deliver these key elements, but in a novel way by holding the consultation outside of a hospital environment and the delivery care led by audiology with remote ENT surgeon input. Patients in the pilot teleotology pathway remained under the care of the hospital throughout the process.

\section{The technology \\ The device}

The TympaSystem (figure 1) is a smartphone-based solution, which combines otoscopy, ear wax removal and hearing screening developed by Tympa Health Technologies Limited (London, UK). The TympaSystem is a Class I medical device, which is CE (Conformité Européenne) marked and compliant with the European Union's Medical Device Regulations under the classification of an otoscope. It is Food Drug Administration approved and $510(\mathrm{k})$ exempt. The device allows for recording of still and video images of a patient's ear through the smartphone's

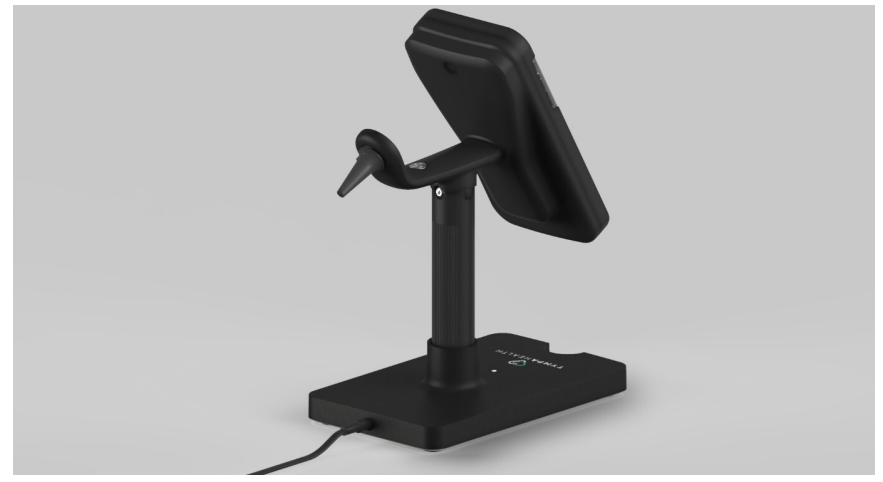

Figure 1 Image of TympaSystem device.

camera and a custom-designed patented optical system. For the purposes of this pilot, only the otoscopy function was used due to uncertainty surrounding microsuction as an aerosol-generating procedure. The audiometry function of the device was not used in this study.

\section{The remote review platform}

TympaHealth has an online software platform that acts as clinic manager, stores data collected by the device (multimedia files) and allows the user to input additional information including history template answers and additional comments. The platform also generates a PDF summary document of all the information generated in the encounter, which can then be shared into the patient record.

\section{Training}

The two audiologists conducting the community-based clinics received a single face-to-face training session with the device and how to use the online platform. They were able to contact the TympaHealth team directly for any technical assistance. Clinicians involved in the remote clinical reviews received a 2-hour evening training session on how to use the remote review platform.

\section{Participants and setting}

Participants were new referrals to otology clinics at a tertiary ENT hospital in central London. Inclusion criteria were adults aged between 18 and 70 with hearing loss or tinnitus. Patients presenting with sudden hearing loss, otalgia, vertigo and otorrhoea were excluded, as were non-English speaking patients. These patients were excluded from the pilot as the listed symptoms were more likely to signify early pathology or pathology that could not be managed via the tele-otology model. NonEnglish speakers were excluded from the pilot due to limitation surrounding face-to-face interpreters during the COVID-19 pandemic. These criteria were set during the COVID-19 pandemic and the age limit of 70 was set due to the UK government shielding criteria. Due to uncertainty surrounding microsuction as an aerosolgenerating procedure, patients with otorrhoea or wax were excluded. These conditions would make evaluation of remote images insufficient due to incomplete views of the tympanic membrane. 


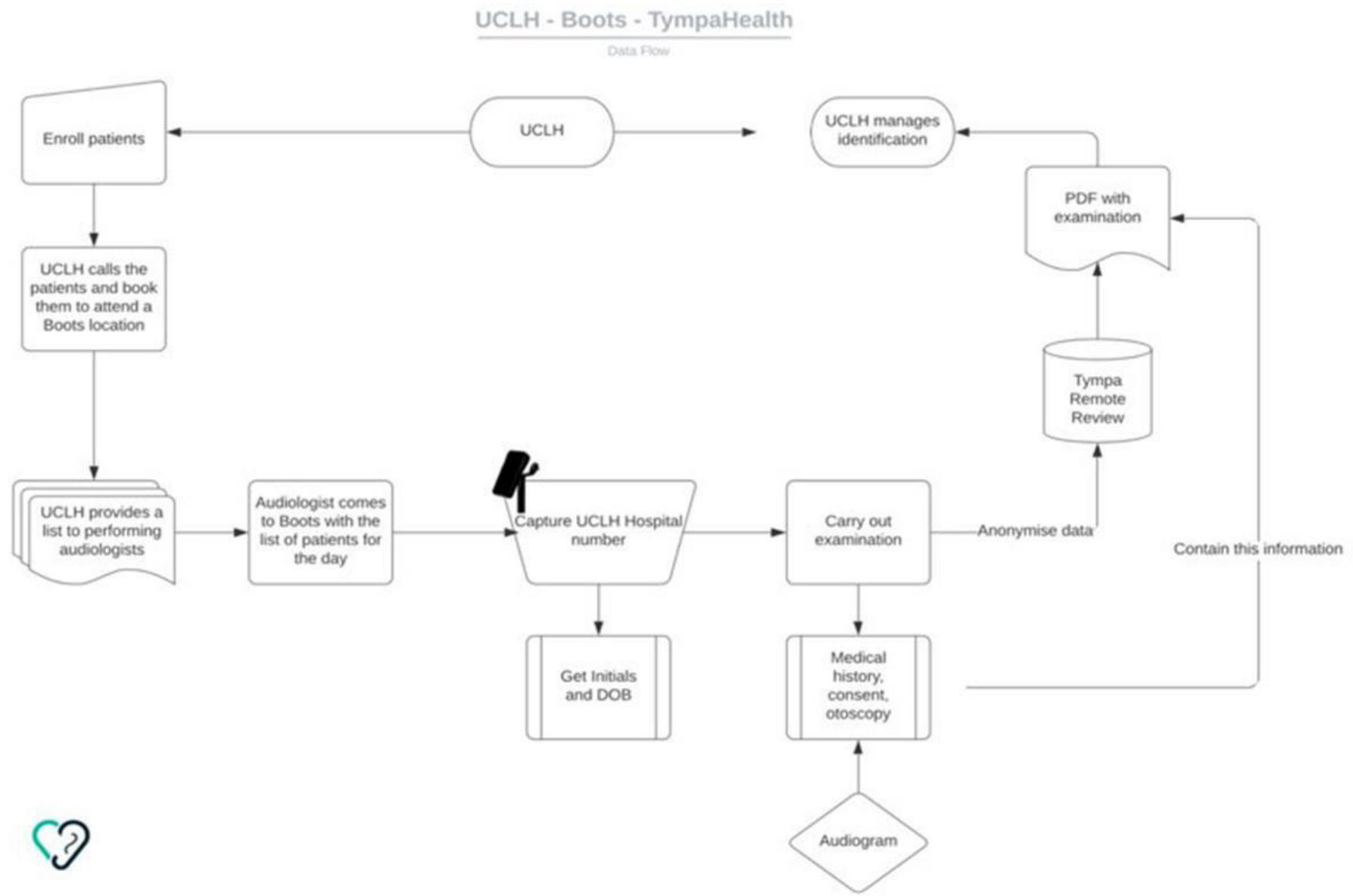

Figure 2 Outline of the teleotology pathway. UCLH, University College London Hospitals; DOB, Date of Birth

Community reviews including audiology testing were undertaken at a Boots Hearingcare site in central London. This site was chosen to simulate a community audiology booth environment offsite from a hospital. Hospital audiology equipment was brought to the location and used for pure tone audiometry and tympanometry; the audiometry feature of the device had not been validated and, therefore, it was not used. Community clinics were delivered by two hospital audiologists. Patients were seen in a 3-week period between 15 July and 6 August 2020.

\section{The pathway}

Initial triage and recruitment

Patients were identified by triaging new referrals to five consultant otologists at our hospital using the information provided in the initial referral letters. Those who met the inclusion criteria were invited to participate by phone or letter. Informed consent was taken verbally and documented in their clinical notes (figure 2).

\section{Community visit}

The audiologist completed a history proforma on the TympaHealth device with the patient (online supplemental appendix 1). The device was then used to examine the patient's ears including both images and video capture. Video capture was performed in order to capture all four quadrants of the tympanic membrane to ensure that the full tympanic membrane could be assessed. The videos were less than $1 \mathrm{~min}$ in duration per ear. If there were no contraindications for further audiological assessment, as determined by the audiologists, diagnostic pure tone audiometry and tympanometry were performed using hospital audiological equipment. Otometrics Aurical AUD S/N: 438482 audiometer and Otometrics Madsen Zodiac S/N 1930136 tympanometer were used, respectively, in accordance with the British Society of Audiologist's recommended procedures (ref 2014, 2018). Air conduction thresholds were measured using insert earphones and bone conduction thresholds using B71 bone transducers.

The results were stored within the hospital electronic medical record Epic (Epic Systems Corporation, USA). Patient satisfaction data were also collected at this time. This entailed completion of a patient questionnaire (online supplemental appendix 2), which assessed a number of aspects of the patient experience of the service using a 10-point Likert scale.

\section{Remote specialist Review}

Three NHS ENT doctors employed at the hospital, the patients were referred to (one consultant, two specialty registrars) remotely reviewed the information collected from the community visit using the TympaHealth platform 
and the patient's electronic health records on the NHS electronic medical record system Epic. Initial review of the patient information was performed by one member of these three doctors prior to a further, blinded validation review by a consultant otologist. The information that was reviewed included the patient history proformas, still and video images of the tympanic membranes, audiometry and tympanometry.

Smart text/templates were created on Epic to standardise the reporting format (online supplemental appendix 3). The templates included information regarding the presence/absence of otological symptoms, the findings from the community visit, a clinical impression of the findings and a management plan. These templates were adapted to the patient-specific information. A letter was sent to the patient and their general practitioner/referring clinician summarising the consultation and management plan. A dedicated email address for the pilot service was included in the letter for patient queries relating to their diagnosis or management.

Following the initial remote review, patients were either discharged from the service, sent for additional investigations, offered telephone follow-up, referred to affiliated departments (audiology or audiovestibular medicine) or given an in-person appointment.

\section{Validation}

In order to assess inter-rater variability, all patients were remotely reviewed by a second consultant otologist who was blind to the initial diagnosis and management plans. Agreement was assessed for diagnosis and management plans.

In order to assess equivalence of the teleotology consultation to a face-to-face consultation, all patients were invited for a face-to-face appointment with a consultant otologist. Diagnosis and management plans were compared with those made by the teleotology pathway and evaluated for concordance and in cases of discordance, the reason for this.

\section{Data analysis}

Descriptive measures were used to analyse the data and the manuscript follows the Standards for QUality Improvement Reporting Excellence (SQUIRE) reporting guidelines.

\section{Patient}

This pathway was developed to allow patients access to specialist review during the acute first wave of the COVID-19 pandemic in the UK, while our hospital-based outpatient clinic was closed to routine clinical activity.

Patients were actively recruited to this study via telephone calls from clinicians involved in the study, explaining the pathway and process of this novel service. They were informed that this was a new pathway being piloted due to the COVID-19 pandemic and was different to the traditional clinical review pathway. They were informed that we would be asking them to complete a patient satisfaction questionnaire at the end of their remote review, where they were asked to assess the novel pathway.

They were aware that they would be offered a faceto-face review at the earliest opportunity, if there was a clinical concern on the remote review. They were also informed that a group of randomly chosen patients would be selected for face-to-face review to validate the findings of the pilot. Patients were also provided with contact details if they had any further comments regarding the pathway or clinical-related queries.

The feedback and comments received from the patients involved in this pilot study will go towards shaping the design of future proposed community-based otology pathways.

\section{RESULTS AND ANALYSIS}

\section{Use of the service}

$23.71 \%$ of all new referrals (243/1029 patients) met the inclusion criteria. $27.05 \%$ of this group agreed to participate in the pilot $(\mathrm{N}=66)$, with $87.87 \%$ attending their appointment $(\mathrm{N}=58)$. Given the pragmatic underpinnings of this study, all patients who agreed to participate were included. Furthermore, the aim of this study was to assess the feasibility of a proposed new service without reference to any one particular end point or outcome measure; therefore, a formal calculation of sample size was not performed. The mean age of participants was 45.2 years (range 20-67) and 60\% were women. Although only new referrals to our hospital were included in this pathway, $67 \%$ of patients had previously been seen by ENT in the past. The demographics and patient characteristics are presented in table 1 .

\section{Community audit data}

Fifty-eight patients were seen in clinic; 93\% (54/58) of those seen had been correctly identified as suitable for inclusion in the pilot based on the agreed inclusion criteria. Of 4 out of 58 patients were found to be unsuitable for inclusion in the pilot study due to wax occlusion or otitis externa. The consultation took $19.4 \mathrm{~min}$ on average and no episodes of patient harm nor adverse events were recorded.

The quality of the combined clinical information (history, examination and audiology tests) was deemed universally adequate for the purposes of the remote clinical review and diagnosis by all reviewers, including the validating otologist. Individual elements in isolation, however, were not always sufficient. Due to the variability in quality and inability to capture the whole tympanic membrane, single still images were not generally adequate to make an assessment of the ear drum compared with the four quadrant still images of the tympanic membrane. For $62 \%$ of patients, the videos captured were considered more suitable for diagnostic purposes compared with the corresponding still images of the tympanic membrane. The videos were felt by the reviewers to provide a more 
Table 1 Demographic data of patients who attended the teleotology clinic

\begin{tabular}{|c|c|c|c|}
\hline Variable & $\mathbf{N}$ & Mean & Range \\
\hline Age (years) & 58 & 45.2 & $20-67$ \\
\hline \multicolumn{4}{|l|}{ Sex } \\
\hline Male & $23(40 \%)$ & & \\
\hline Female & $35(60 \%)$ & & \\
\hline \multicolumn{4}{|l|}{ Occupation } \\
\hline Professional other & 10 & & \\
\hline Education & 8 & & \\
\hline Skilled technical & 7 & & \\
\hline Health & 5 & & \\
\hline Unemployed & 5 & & \\
\hline Caring/leisure services & 4 & & \\
\hline Retired & 3 & & \\
\hline Sales & 3 & & \\
\hline Machine operatives & 2 & & \\
\hline Administrator & 2 & & \\
\hline Skilled trade & 2 & & \\
\hline Social care & 2 & & \\
\hline Charity/ volunteer & 2 & & \\
\hline \multicolumn{4}{|l|}{ Previously seen by ENT } \\
\hline Yes & 39 & & \\
\hline No & 16 & & \\
\hline \multicolumn{4}{|l|}{ Diagnosis } \\
\hline Presbycusis & 1 & & \\
\hline Mixed loss & 2 & & \\
\hline Normal & 4 & & \\
\hline Asymmetrical SNHL & 12 & & \\
\hline Otitis externa & 1 & & \\
\hline Perforation & 5 & & \\
\hline Unilateral OME & 5 & & \\
\hline Otosclerosis & 4 & & \\
\hline $\begin{array}{l}\text { Tympanic membrane } \\
\text { retracted }\end{array}$ & 2 & & \\
\hline Tinnitus & 9 & & \\
\hline Eustachian tube dysfunction & 5 & & \\
\hline Auditory processing disorder & 1 & & \\
\hline Noise-induced hearing loss & 1 & & \\
\hline Symmetrical SNHL & 2 & & \\
\hline Cholesteatoma & 2 & & \\
\hline Conductive hearing loss & 1 & & \\
\hline Non-organic hearing loss & 1 & & \\
\hline
\end{tabular}

ENT, ear, nose and throat; OME, Otitis Media with effusion; SNHL, Sensorineural hearing loss.

dynamic assessment of the tympanic membrane akin to microscopic evaluation in the ENT clinic setting. Factors contributing to poor image and video quality were
Table 2 Summary of patient outcomes, onward referrals and imaging requests in the teleotology pilot

\begin{tabular}{lc}
\hline Variable & Result \\
\hline Attended appointment & 58 \\
\hline Did not attend (n) & 8 \\
\hline Time per patient review (min) & 10.3 \\
\hline Outcomes ( $\mathrm{n})$ & \\
\hline Discharged & $19(32.8 \%)$ \\
\hline Face-to-face appointment & $20(34.5 \%)$ \\
\hline Video consultation & $10(17.2 \%)$ \\
\hline Letter with results & $9(15.5 \%)$ \\
\hline Imaging requests $(\mathrm{n})$ & 27 \\
\hline CT temporal bone & 6 \\
\hline Cone beam CT & 3 \\
\hline MRI & 18 \\
Onward referrals (n) & 21 \\
\hline Hearing aid & $14(24.1 \%)$ \\
\hline Audiovestibular medicine & $3(5.1 \%)$ \\
\hline Tinnitus therapy & $6(10.3 \%)$ \\
\hline
\end{tabular}

obstruction of views by wax, incomplete visualisation of the entire tympanic membrane and inadequate focus.

\section{Patient outcomes}

Table 2 summarises the main patient outcomes following the initial teleotology clinic appointment. Nearly, onethird of patients were discharged following the community clinic. Just over $15 \%$ of patients had no organised follow-up but received a letter with imaging results and a further $17.2 \%$ had teleconsult follow-up.

Of the 20 patients requiring further review, six of these appointments did not result in a lengthened pathway as they required further in-person reviews following imaging requests and, therefore, would have also been required if the patients had been seen face-to-face initially. Half of the 14 patients with lengthened clinical pathways (7) required further examination for possible unilateral otitis media with effusion, including examination of the postnasal space. A management plan could not be made in $5 / 14$ due to inadequate views of the ear drum, and a 2/14 had conditions identified that were not suitable for the remit of the pilot study.

Therefore, our results show that $75.8 \%(\mathrm{~N}=44)$ of our samples of of patients had their pathway shorted by one hospital visit and $65.6 \%$ patients avoided any hospital attendances for review in clinic.

\section{Validation}

All 58 patients were reviewed by a blinded consultant. Concordance with diagnosis between the blinded consultant otologist reviewing the remote reviews was 94.8\%. Modifications of diagnosis included a false fundus, Iincudostapedial joint erosion and possible hydrops; despite this, there was only one patient who required a 
Table 3 Reasons for disagreement with management plans by validating consultant otologist

\begin{tabular}{ll}
\hline Reason & Frequency \\
\hline Imaging & 3 \\
\hline Additional scans & 2 \\
Unnecessary scans & \\
Onward referral & 1 \\
$\quad$ Hearing aid & 1 \\
Advanced audiology & \\
Follow-up modality & 1 \\
Face-to-face required & 1 \\
\hline Treatment & 9 \\
\hline Total & \\
\hline
\end{tabular}

change to their management plan. Management plans were agreed in $84.5 \%$ of patients. Reasons for disagreements are summarised in table 3 .

Twelve of the 58 patients agreed to attend hospital for an in-person review. There was agreement with the diagnosis and management plans between consultant-remote review and in-person consultant review in $83.3 \%$ of cases $(10 / 12)$ with some minor modifications to management plans made at the face-to-face visits. These included the addition of a CT scan request for a patient with bilateral false fundus and a second case involved not recommending a hearing aid.

\section{Patient satisfaction}

Ninety-eight per cent of patients stated that they would recommend the service to another patient and $76.8 \%$ of patients were happy being seen by an audiologist and would not have preferred being seen by a doctor. Average satisfaction scores out of 10 were as follows: 8.52 for waiting time for the appointment, 9.91 for waiting time on the day of the appointment, 9.96 for appointment length and 8.98 for business of the waiting area.

\section{DISCUSSION}

Footfall reduction in the hospital is pressing in view of the ongoing threat from COVID-19. Even before this novel pathogen, hospital outpatient clinics were overwhelmed by increasing patient numbers who faced ever-growing delays to be seen.

Remote review services offer an alternative pathway for the assessment and management of patients with otological reports in the community. Nearly, two-thirds of patients in our cohort avoided attending a hospital-based appointment.

Diagnostic and management concordance are incredibly important to ensure a safe, non-inferior service to the traditional consultation model. Diagnostic concordance with a blinded consultant otologist reviewing the remote reviews was $94.8 \%$, and $83.3 \%$ in randomly selected in-person reviews. Similarly, management plans were agreed in $84.5 \%$ of patients with blinded remote reviews and $83.3 \%$ of the randomly selected face-to-face reviews. These figures correlate well with a recent systematic review of diagnostic concordance in otolaryngology telemedicine. ${ }^{10}$ They concluded that an asynchronous review model did not suffer from reduced diagnostic concordance, particularly when there is an adequate level of clinical information provided, which we ensured in our pilot through the use of audiology-led history and examinations.

A key consideration in developing this service is case selection. As our triaging criteria were retrofitted to the new referrals for the purposes of the pilot, a dedicated teleotology referral criterion would reduce the inappropriately triaged patient numbers and subsequent lengthened pathways.

In our pilot, we limited the service to patients with hearing loss and/or tinnitus. Otorrhoea was excluded given the uncertainty in the early days of the pandemic surrounding classification of aerosol-generating procedures. $^{11} 12$ The device itself can facilitate suction clearance of the ear canal and, with training, practitioners using this device to suction could see a broader patient group as well as reducing hospital visits for microsuction for wax removal.

Furthermore, if wax removal had taken place in our pilot, this would have reduced the number of patients needing to reattend. Wax removal is a simple intervention that can have alleviate a reversible cause of temporary deafness, which is associated with significant impairment of quality in life and threats to safety. The NICE Quality Standards for Hearing Loss in Adults (National Institute for Health and Care Excellence) irecommends that wax removal should be performed in primary care or community ear care services, to prevent the inappropriate use of specialist services. ${ }^{13}$ However, at the same time, a greater number of commissioning groups have cut this essential service.

Undoubtedly, using the device for procedures such as suctioning, will require additional training to ensure safe and effective performance. This, however, is an easily surmountable obstacle with additional training. Indeed, in many centres, microsuction clinics are nurse led.

We have also demonstrated that teleotology can be used to list patients for surgery. This supports previous work, where telemedicine surgical recommendations were found to match recommendations following in-person reviews for a series of 45 patients who were seen independently in both settings. ${ }^{14}$

Our community review was led by audiologists. This professional group are well-placed to provide such a service, particularly as audiologists are experienced in ear examination, history taking and audio-vestibular testing. Indeed, audiologist-led store-and-forward telemedicine consultations have been successful in rural Alaska, where they were associated with a $50 \%$ reduction in waiting times to be seen by ENT across a 6 -year period. ${ }^{15}$ This reduction was attributed to the fact that most referrals 
could be managed within the audiology-led clinic, thus increasing availability of clinic slots for others. Our study corroborates this given that a significant proportion of the complaints were managed within the single audiology-led consultation or through subsequent referral to audiology led clinics (tinnitus therapy and hearing aid centre). The delivery of this service, however, could be broadened further. With suitable training, other healthcare practitioners could facilitate such pathways.

In addition to a community-based audiology clinic for ontological reports, there are alternative models that our pathway could be applied to. Once such example is the development of an advice-and-guidance service for GPs. This could reduce patient referrals to the hospital due to triage by the remotely reviewing specialists. This model has been described previously, but the smart-otoscope used here is likely to be more accessible to either an audiology or GP-led service due to instrument familiarity over an otoendoscope used in other pilots. ${ }^{3}$ The Tympa system also benefits from the online secure platform, which was accessible and user-friendly, facilitating a store-andforward platform and asynchronous remote specialist review. This platform produces a patient record that can be incorporated into the patients' medical records.

A further application could be for audiology-led postoperative cholesteatoma surveillance clinics with specialist review of any concerning findings. This again represents another potential use to reduce the burden on specialist clinics. Khalid-Raja et al analysed hospital episode statistics data to estimate that, over the four selected years between 2005 and 2012, an average of 5074 patients underwent operations for cholesteatoma per year. ${ }^{16}$ Using the same Office of Population Censuses and Surveys Classification of Interventions and Procedures codes, 4635 patients had similar surgery in 2019. ${ }^{17}$ These patients are routinely followed up for a number of years; therefore, clinical surveillance can represent a significant burden in otology clinics.

Our pilot study is not without limitations. First, the sample size of patients is small. Of those eligible, just under one quarter of patients agreed to participate. Our sample, therefore, may not be sufficiently powered to be representative of the wider population who would be eligible for assessment in such a service in the future. As already discussed, with the use of microsuction functions of the device, the inclusion criteria could be broadened to include patients with wax impaction and/or discharge. Second, only a proportion (12/58) of the patients underwent in-person clinical validation. Given the current public health concerns surrounding coronavirus at the time the study was undertaken, it is perhaps unsurprising that patients did not want to be seen in a hospital setting. Future studies will benefit from a greater proportion of patients being reviewed in person to assess concordance of examination findings, diagnosis and management plans. Finally, capture of clear views of the tympanic membrane was challenging in patients with narrow or tortuous ear canals. Video capture was able to get around this problem by providing a dynamic view of the whole drum. Further work has been suggested to increase the range of speculum sizes to account for variations in ear canal anatomy in order to optimise visibility.

Future development of this pathway would also benefit from thle use of a validated measure of patient satisfaction.

\section{Conclusion}

We have demonstrated that this innovative tele-otology service model is clinically safe and non-inferior to the traditional clinical model.

Hospital pathways were shortened in the vast majority of patients, and hospital footfall reduced by delivering care in the community and arranging follow-up via telephone or video consultations. In an appropriately structured service, cost reductions from community-led care should be expected. Despite the remote management, the patient experience did not suffer as $98 \%$ of patients would recommend the service to others. With triage criteria improvements and use of microsuction, further reductions in pathway length and hospital footfall should be expected.

Looking ahead, the teleotology model could be applied to audiology-led community clinics such as our model, GP advice-and-guidance services or audiology-led cholesteatoma surveillance clinics.

Acknowledgements With thanks to Helen Fulbright PhD, MA, PGDip, BA (Hons), Information Specialist, Royal College of Surgeons of England Library and Archives Team, for conducting the literature searches; Dominic Firth \& Lucy Pickup from the Commercial \& Contracts Team at UCLH; Matthew Hall from the Information Governance and Data Protection Team at UCLH; Robert Skedge and Karen Shephard from Boots Hearingcare; and Krishan Ramdoo and the TympaHealth Team.

Contributors CTF and LD wrote the initial draft and analysed the results with input from the senior authors; they contributed equally to the project. SD, JP and $\mathrm{DC}$ contributed to the audiological aspects of the study and those aspects of the final paper. MB, NM and JGM were involved in the overall design of the study and contributed to the final paper. All authors approved the final submitted manuscript. $\mathrm{JM}$ is responsible for the overall content as the guarantor.

Funding The pilot launch was made possible by non-commercial donation of clinic rooms by Boots Hearingcare, whilst NHS capacity was restricted. Boots made facilities available in their Oxford Street Store on a non-commercial basis as a gesture of goodwill towards the NHS. A memorandum of understanding was agreed between the hospital Trust and Boots Hearingcare. This was signed by the Trust's legal team and data protection impact assessment was signed off by the Trust information Governance Team. Tympa Health provided devices, training and access to their remote review platform on a cost-neutral basis for the purpose of this pilot.

Competing interests None declared.

Patient consent for publication Not applicable.

Ethics approval Formal ethical approval was not needed to assess the feasibility of this pilot service reconfiguration. The TympaHealth device is CE marked and compliant with the EU Regulations for medical devices. A memorandum of understanding was agreed between the hospital Trust and Boots Hearingcare. This was agreed by the Trust's legal team and a data protection impact assessment was agreed by the Trust's information Governance team.

Provenance and peer review Not commissioned; externally peer reviewed.

Data availability statement Data are available upon reasonable request. A data protection impact assessment was agreed by the Trust's information Governance team. The data are held in the UCLH eletronic health records and the Tympa Health secure platform. Due to confidentialty of personal patient data, data are not available on request. 
Supplemental material This content has been supplied by the author(s). It has not been vetted by BMJ Publishing Group Limited (BMJ) and may not have been peer-reviewed. Any opinions or recommendations discussed are solely those of the author(s) and are not endorsed by BMJ. BMJ disclaims all liability and responsibility arising from any reliance placed on the content. Where the content includes any translated material, BMJ does not warrant the accuracy and reliability of the translations (including but not limited to local regulations, clinical guidelines, terminology, drug names and drug dosages), and is not responsible for any error and/or omissions arising from translation and adaptation or otherwise.

Open access This is an open access article distributed in accordance with the Creative Commons Attribution Non Commercial (CC BY-NC 4.0) license, which permits others to distribute, remix, adapt, build upon this work non-commercially, and license their derivative works on different terms, provided the original work is properly cited, appropriate credit is given, any changes made indicated, and the use is non-commercial. See: http://creativecommons.org/licenses/by-nc/4.0/.

ORCID iDs

Cillian T Forde http://orcid.org/0000-0003-2017-2056

Lilia Dimitrov http://orcid.org/0000-0002-3959-7292

\section{REFERENCES}

1 NHS Long Term Plan » Online version of the NHS Long Term Plan. Available: https://www.longtermplan.nhs.uk/online-version/ [Accessed 8 Sep 2020].

2 NHS Digitial. Hospital Outpatient Activity 2018-19 - NHS Digital, 2019. Available: https://digital.nhs.uk/data-and-information/ publications/statistical/hospital-outpatient-activity/2018-19 [Accessed 8 Sep 2020].

3 Cottrell E, George A, Coulson C, et al. Telescopic otology referrals: evaluation of feasibility and acceptability. Laryngoscope Investig Otolaryngol 2020;5:221-7.

4 Eikelboom RH, Atlas MD, Mbao MN, et al. Tele-otology: planning, design, development and implementation. J Telemed Telecare 2002;8 Suppl 3:14-17.

5 Mandavia R, Lapa T, Smith M, et al. A cross-sectional evaluation of the validity of a smartphone otoscopy device in screening for ear disease in Nepal. Clin Otolaryngol 2018;43:31-8.
6 Demant MN, Jensen RG, Bhutta MF, et al. Smartphone otoscopy by non-specialist health workers in rural Greenland: a cross-sectional study. Int J Pediatr Otorhinolaryngol 2019;126:109628.

7 Elliott G, Smith AC, Bensink ME, et al. The feasibility of a communitybased mobile telehealth screening service for Aboriginal and Torres Strait Islander children in Australia. Telemed J E Health 2010;16:950-6.

8 ENT UK. Guidance for ENT during the COVID-19 pandemic, 2020. Available: https://www.entuk.org/guidance-ent-during-covid-19pandemic [Accessed 30 Sep 2020].

9 Meng X, Dai Z, Hang C, et al. Smartphone-enabled wireless otoscope-assisted online telemedicine during the COVID-19 outbreak. Am J Otolaryngol 2020;41:102476.

10 Ning AY, Cabrera Cl, D'Anza B, D'Anza B. Telemedicine in otolaryngology: a systematic review of image quality, diagnostic concordance, and patient and provider satisfaction. Ann Otol Rhinol Laryngol 2021;130:195-204.

11 Philpott C, Burrows S. Aerosol-generating procedures in ENT. ENT UK at the Royal College of Surgeons of England, 2020. Available: https://www.entuk.org/aerosol-generating-procedures-ent [Accessed 30 Sep 2020].

12 Rea P, Lloyd S, Jenkins D. Guidance for undertaking otological procedures during COVID-19 pandemic. British Society of Otology, 2020. Available: https://www.entuk.org/guidance-undertakingotological-procedures-during-covid-19-pandemic [Accessed $30 \mathrm{Sep}$ 2020].

13 National Institute for Health and Care Excellence. Quality statement 1: Earwax removal | hearing loss in adults | quality Standards | NICE. NICE, 2019. Available: https://www.nice.org.uk/guidance/qs185/ chapter/Quality-statement-1-Earwax-removal [Accessed 22 Sep 2020].

14 Kokesh J, Ferguson AS, Patricoski C. Preoperative planning for ear surgery using store-and-forward telemedicine. Otolaryngol Head Neck Surg 2010;143:253-7.

15 Hofstetter PJ, Kokesh J, Ferguson AS, et al. The impact of telehealth on wait time for ENT specialty care. Telemed J E Health 2010;16:551-6.

16 Khalid-Raja M, Tikka T, Coulson C. Cholesteatoma: a disease of the poor (socially deprived)? Eur Arch Otorhinolaryngol 2015;272:2799-805.

17 NHS Digital. Hospital Admitted Patient Care Activity 2018-19 - NHS Digital, 2019. Available: https://digital.nhs.uk/data-and-information/ publications/statistical/hospital-admitted-patient-care-activity/2018 19 [Accessed 30 Sep 2020]. 